DOI: https://doi.org/10.29393/EID3-9DELG400o9

\title{
DISEÑO ERGONÓMICO DE UNA BUTACA PARA ESTUDIANTES DE INGENIERÍA MECÁNICA
}

\author{
ERGONOMIC DESIGN OF AN ARMCHAIR FOR STUDENTS OF MECHANICAL \\ ENGINEERING
}

\author{
Litzy Jaquelin, Gutiérrez Vázquez \\ Ana Isabel, Ramos Cortez ${ }^{* *}$ \\ Xóchitl Patricia, Flores Gutiérrez ${ }^{* * *}$ \\ Yuridia Belén, Cota Pardini ${ }^{* * * *}$
}

\begin{abstract}
Resumen: Los estudiantes de ingeniería mecánica están en las aulas y laboratorios del campus durante aproximadamente siete horas diarias, utilizando butacas que no han sido diseñadas ergonómicamente y que los obligan a adoptar posturas incorrectas durante la jornada escolar. Sabiendo que una butaca ergonómica supone cuestiones de salud, mayor confort y, por ende, mayor energía y motivación, en esta investigación, el objetivo fue el diseño ergonómico de una butaca para estudiantes específicamente de este perfil en una universidad. Para diseñar la butaca, se desarrolló un estudio antropométrico a los estudiantes de la carrera con edades entre los 18 y 22 años y se elaboró una carta antropométrica con las dimensiones requeridas. Considerando una población de 100 de alumnos de la carrera, se determinó el tamaño ideal de la muestra que represente al percentil 95. Las dimensiones estructurales de los diferentes segmentos del cuerpo se tomaron en individuos con posturas estáticas de pie o sentado utilizando instrumentos como antropómetro, flexómetro digital, balanza digital y talímetro, dando como resultado el diseño ergonómico de una butaca escolar.
\end{abstract}

Palabras clave: Ergonomía, antropometría, percentil, dimensión, postura.

\begin{abstract}
The students of Mechanical Engineering are in the campus classrooms and laboratories for approximately 7 hours every day. Utilizing armchairs that have not been ergonomically designed and which forces them to adopt incorrect postures during the school day. Knowing that an ergonomic armchair supposes health issues, greater comfort, and therefore, much energy and motivation, in this research, the aim was the ergonomic design of an armchair for students specifically from this profile in an university. To design the armchair, developed an anthropometric study to the students of the career with ages between 18 and 22 years, and an anthropometric chart was made with the required dimensions. Considering a population of 100 students of the career, it was determined the ideal size of the sample to include a percentile of $95 \%$. The structural dimensions of the different body segments were taken on individuals with standing or seated static postures, like anthropometer, digital

\footnotetext{
"Instituto Tecnológico Superior de Guasave. Guasave, México. Correo electrónico: Jaquelin.2ocbtis@gmail.com. Orcid: https://orcid.org/oooo-0002-3526-6939

** Instituto Tecnológico Superior de Guasave. Guasave, México. Correo electrónico: ana_raco99@hotmail.com. Orcid: https://orcid.org/oooo-0002-0947-0119

***Instituto Tecnológico Superior de Guasave. Guasave, México. Correo electrónico: xochitl.fg@guasave.tecnm.mx. Orcid: https://orcid.org/oooo-0oo1-6468-3865

*****Instituto Tecnológico Superior de Guasave. Guasave, México. Correo electrónico: Ing.belencota@gmail.com. Orcid: https://orcid.org/oooo-0003-1962-9274. Autor de correspondencia.
} 
flexometer, digital balance, and height rod, resulting in an ergonomic design of a school chair that reduces aforementioned problems and consequently contributes to academic performance.

Keywords: Ergonomics, anthropometric, percentile, dimension, posture.

Recepción: 21.04.2021 / Revisión: 23.04.2021 / Aceptación: 04.05.2021

\section{Introducción}

La ergonomía se define como el campo de conocimientos multidisciplinar que estudia las características del ser humano con la finalidad de adaptar los trabajos y los productos a sus capacidades y necesidades (Vergara \& Agost, 2015). La aplicación de la ergonomía tiene como objetivo adaptar productos, herramientas y áreas de trabajo, con la finalidad de mejorar el bienestar de los usuarios. En instituciones universitarias, es común observar a estudiantes que no consiguen sustentar sus pies en la superficie debido a su baja estatura, o bien aquellos cuyas dimensiones de las extremidades inferiores impiden adoptar una buena postura, ya que el espacio disponible es insuficiente. Sin duda, el disconfort de los estudiantes es una variable que repercute en el desarrollo de las actividades educativas, ya que un mobiliario inadecuado impide que la ejecución de las tareas sea favorable.

Medina et al. (2011) señalan que países como Brasil, Colombia, Estados Unidos y Venezuela han realizado investigaciones sobre las condiciones disergonómicas en las aulas de clase en distintas universidades nacionales. En este sentido, Rodríguez y González (2011) hacen una compilación de la evolución del mobiliario escolar para evaluar si este está siguiendo parámetros ergonómicos e indican que la adaptación del mobiliario escolar a la antropometría o a las necesidades físicas de los niños es el motivo principal de los avances más relevantes; sin embargo, explican que esta evolución no siempre ha sido positiva, desde el punto de vista ergonómico. Concluyen que una de las preocupaciones que se plantean hoy en día es la falta de adaptación del mobiliario y la incomodidad que puede generar y que las características del mobiliario escolar tradicional obligan a los estudiantes a adoptar posiciones anti fisiológicas, que con el tiempo pueden tener como consecuencia graves problemas de salud.

Para hacer un adecuado diseño ergonómico, la antropometría es fundamental. El término antropometría proviene del griego anthropos (hombre) y metrikos (medida) y trata del estudio cuantitativo de las características físicas del hombre. Existen muchos autores que señalan a la antropometría como un conjunto de técnicas derivadas de la antropología física. La antropometría es la ciencia que estudia las dimensiones y proporciones del cuerpo humano. Los datos antropométricos son necesarios para establecer las dimensiones de los productos y espacios de trabajo. Se aplica en todos los ámbitos del diseño industrial (Vergara \& Agost, 2015). Puesto que la antropometría aplicada al diseño es el uso de distintos métodos e instrumentos validados de mediciones físicas de una población específica, el resultado de las medidas ayuda a dimensionar el prototipo que se desea diseñar y ayudan a establecer estrategias para saber cómo se ajustará a las dimensiones de la población (Breve introducción a la antropometría física, 2010, 10 de marzo). 
Ávila et al. (2007) consideran que las dimensiones antropométricas de cada población son denominadas por medidas y se definen como percentiles, los cuales son valores que dividen un conjunto de datos estadísticos en forma de porcentaje que sea inferior a los distintos valores obtenidos. Existen muchos factores que intervienen significativamente en las dimensiones corporales de los seres humanos, como lo pueden ser el sexo, la edad, raza, etc. Una de las diferencias más significativas se encuentra entre hombres y mujeres, ya que por lo general las personas del sexo masculino suelen tener dimensiones corporales mayores que las personas del sexo femenino. Las mediciones tomadas deben ser lo más exactas posibles, para que el estudio tenga la confiabilidad necesaria para su aplicación al diseño de objetos o elementos de uso común (Ruiz, 2008).

Esta investigación parte de recabar las medidas antropométricas de la población estudiantil de ingeniería mecánica, con el objetivo de diseñar una butaca que se adapte a sus características.

\section{Materiales y métodos}

\section{Diseño de carta antropométrica}

Se realizó un estudio antropométrico en el laboratorio de estudio de trabajo y ergonomía de la carrera de ingeniería industrial en una universidad, con una muestra de alumnos de la carrera de ingeniería mecánica, con la intención de calcular el percentil 5 y 95, para diseñar una butaca ergonómica para la población actual de dicha carrera.

Para seleccionar la muestra se determinaron las medidas que se necesitaban considerar para dicho diseño, y se codificaron para identificarlas en cada uno de los sujetos.

Figura 1. Carta antropométrica.

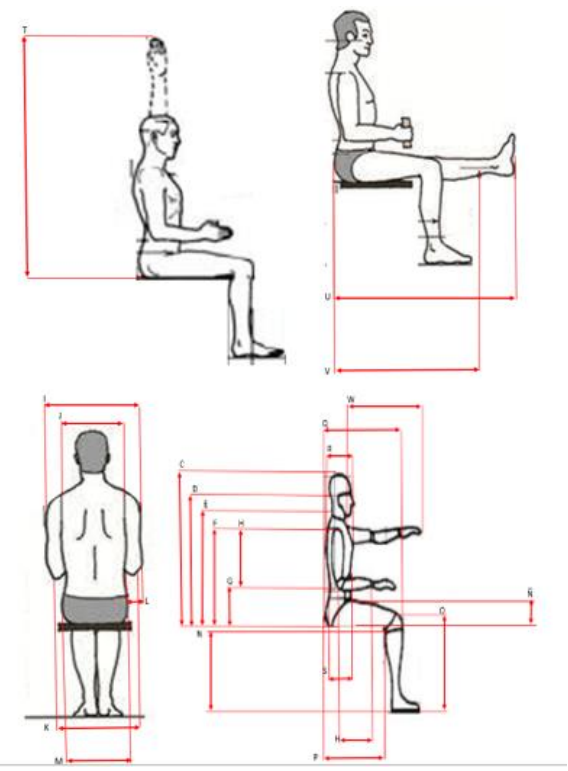


Tabla 1. Dimensiones seleccionadas y sus códigos.

\begin{tabular}{|l|c|}
\hline \multicolumn{1}{|c|}{ Dimensión } & Código \\
\hline Peso & $\mathrm{A}$ \\
\hline Altura de pie & $\mathrm{B}$ \\
\hline Altura sentado & $\mathrm{C}$ \\
\hline Altura de los ojos (sujeto sentado) & $\mathrm{D}$ \\
\hline Altura de la nuca (sujeto sentado) & $\mathrm{E}$ \\
\hline Altura de los hombros (sujeto sentado) & $\mathrm{F}$ \\
\hline Altura del codo asiento (sujeto sentado) & $\mathrm{G}$ \\
\hline Longitud hombro codo (sujeto sentado) & $\mathrm{H}$ \\
\hline Longitud codo muñeca (sujeto sentado) & $\mathrm{H}$ \\
\hline Anchura de hombros 2 (anchura bideltoidea, sujeto sentado) & $\mathrm{I}$ \\
\hline Anchura de hombros (sujeto sentado) & $\mathrm{J}$ \\
\hline Anchura entre codos (sujeto sentado) & $\mathrm{K}$ \\
\hline Anchura del codo (sujeto sentado) & $\mathrm{L}$ \\
\hline Anchura de caderas (sujeto sentado) & $\mathrm{M}$ \\
\hline Altura del poplíteo (sujeto sentado) & $\mathrm{N}$ \\
\hline Espesor del muslo (sujeto sentado) & N \\
\hline Altura de la rodilla (sujeto sentado) & $\mathrm{O}$ \\
\hline Longitud poplíteo trasero (sujeto sentado) & $\mathrm{P}$ \\
\hline Longitud rodilla trasero (sujeto sentado) & $\mathrm{Q}$ \\
\hline Espesor del pecho P (profundidad del pecho, sujeto sentado) & $\mathrm{R}$ \\
\hline Espesor abdominal (sujeto sentado) & $\mathrm{S}$ \\
\hline Altura alcance vertical (sujeto sentado) & $\mathrm{T}$ \\
\hline Distancia nalga punta del pie (sujeto sentado) & $\mathrm{U}$ \\
\hline Distancia nalga pierna (sujeto sentado) & $\mathrm{V}$ \\
\hline Alcance punta dedo (sujeto sentado) & $\mathrm{W}$ \\
\hline
\end{tabular}

\section{Cálculo del tamaño de la muestra}

Para determinar el tamaño de la muestra se consideró una población de 100 estudiantes de la carrera de ingeniería mecánica y se calculó el tamaño de la muestra, dando un total de 43 alumnos que se eligieron de forma aleatoria.

$$
n=\frac{z^{2} * \mathrm{p} * \mathrm{q} * \mathrm{~N}}{e^{2}(\mathrm{~N}-1)+z^{2} * \mathrm{p} * \mathrm{q}}
$$

Donde:

$\mathrm{N}=$ total de la población.

Z2 = 1.962 (si la confiabilidad es del 95\%).

p y q = proporción esperada 0,5 , para una máxima probabilidad de cada sujeto de ser tomada en cuenta.

e: precisión. 


$$
n=\frac{196^{2} * 0.95 * 0.05 * 100}{0.05^{2}(100-1)+\left(1.96^{2} * 0.95 * 0.05\right)}=42.44 \approx 43
$$

\section{Toma de mediciones antropométricas}

Las medidas se realizaron dentro de las instalaciones del laboratorio, donde los alumnos acudieron durante cuatro días consecutivos tomando las siguientes consideraciones:

1) El espacio cuenta con las condiciones de confort adecuado, buena iluminación, espacio amplio y temperatura confortable.

2) Los sujetos estudiados estaban descalzos y con ropa ligera.

3) Tomando en cuenta que el peso corporal y estatura sufren variaciones a lo largo del día, las medidas se realizaron a la primera hora del día entre las 7 y 9 am, con recomendaciones de alimentación previas.

4) Los instrumentos fueron calibrados y comprobada su exactitud antes de realizar las mediciones.

5) Se marcaron en la carta antropométrica previamente los puntos anatómicos y de referencia.

Para realizar las mediciones se utilizaron los instrumentos disponibles en el laboratorio, tales como flexómetro, talímetro, antropómetro y balanza digital.

Tabla 2. Instrumentos utilizados en las mediciones.

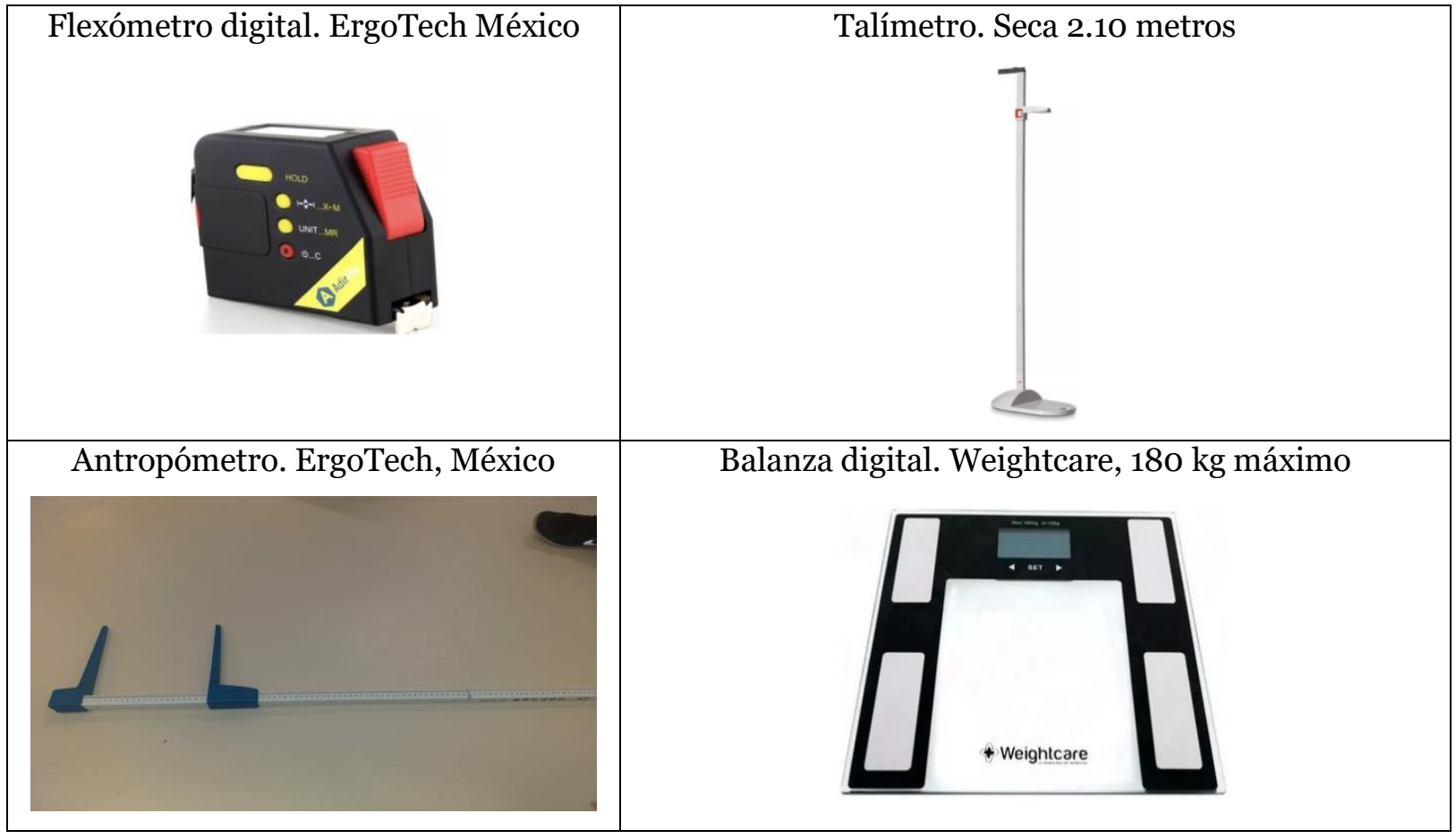

Los datos obtenidos de las mediciones, fueron almacenados y procesados en hojas de cálculo en Microsoft Excel para determinar los percentiles. Con el objetivo de que un mayor número de individuos se encuentre dentro de una dimensión corporal igual o menor a las 
determinadas en el estudio, se eligió el percentil del 95.

\section{Diseño de butaca ergonómica}

A partir de los datos obtenidos con las mediciones, se calcularon los percentiles 5 y 95 en el programa Excel para determinar las medidas adecuadas para el diseño de la butaca ergonómica, para los estudiantes de la carrera de ingeniería mecánica. Se utilizó SolidWorks 2014 para hacer el diseño de la butaca.

\section{Resultados y discusión}

Cervantes-Soto et al. (2019) desarrollaron un diagnóstico de lumbalgia en estudiantes universitarios del área de salud en Tepic, Nayarit donde participaron 90 estudiantes de la licenciatura en nutrición y fisioterapia (45 de cada profesión). Los estudiantes de nutrición fueron quienes presentaron mayor problema, el 77,77\% manifestó dolor de espalda. Aunque las posturas inadecuadas fueron un factor predominante para padecer dolor lumbar, señalan que la percepción dolorosa en la zona lumbar se debió a las malas medidas ergonómicas del mobiliario y butacas, que no se adaptan a la complexión de la mayoría de los estudiantes.

Por su parte, Párraga y García (2014) identificaron los factores que influyen en la incomodidad del estudiante universitario de la facultad de ingeniería industrial de la Universidad Nacional Mayor de San Marcos (UNMSM), señalando que el aspecto ergonómico que más influye en la incomodidad de los estudiantes universitarios es el mobiliario, principalmente el asiento de la carpeta debido a la dureza del material. Esto ocasiona que, al final de la jornada, los estudiantes manifiesten malestar en las sentaderas y, en menor medida, en los muslos y espalda media. Concluye que definitivamente, las medidas del mobiliario del aula no guardan relación con las medidas antropométricas de los estudiantes.

Como ya se ha hecho mención, el objetivo de esta investigación fue el diseño de una butaca que cumpla con las dimensiones apropiadas para que el 95\% de los alumnos de la carrera de ingeniería mecánica adopte una postura adecuada que le permita cuidar su salud y mantener el rendimiento académico. Es importante mencionar que actualmente la institución cuenta con una butaca clásica que no cumple con los estándares ergonómicos requeridos.

La Unión General de Trabajadores (s.f) menciona que una de las funciones principales de la ergonomía es la adaptación de los puestos de trabajo. En esta investigación se realizó un análisis ergonómico dirigido a la elaboración de las butacas, utilizando la antropometría, con el fin de determinar los factores que influyen y cuáles deben ser los valores ideales para conseguir el confort y a su vez la eficacia del trabajo que se realice.

En los puestos de trabajo, la naturalidad de movimientos y posturas son cruciales para el buen desempeño y la salud de las personas, considerando que el puesto se adapte a las dimensiones corporales de los estudiantes de la carrera, no dejando de lado las diversas variedades que existen entre las tallas de los estudiantes a evaluar, para obtener la butaca 
ergonómica.

Para lograr el diseño ideal de los puestos de trabajo, es más lógico y correcto tomar en cuenta a los individuos de mayor estatura para poder acotar las dimensiones, un ejemplo claro sería la distancia de las piernas debajo de la butaca, por otra parte, también considerando a los individuos de una estatura inferior, para acotar dimensiones de las zonas de alcance. Los percentiles ideales serían el 5 y 95.

Debido a que el percentil 5 y 95 corresponden a los porcentajes de la población que se encuentra en el valor de la dimensión o por debajo, en una distribución normal, estos dos puntos son simétricos respecto a la media, es decir, las dos medidas se ubican a igual distancia de la media.

En el anexo, se muestra en detalle el registro de las 25 mediciones tomadas a 43 estudiantes del campus y, a continuación, la tabla 3 ilustra los cálculos de los percentiles 5 y 95 .

Tabla 3. Cálculo de percentiles.

\begin{tabular}{|l|c|c|}
\hline \multicolumn{1}{|c|}{ Dimensión } & Percentil $\mathbf{5}^{*}$ & Percentil 95 \\
\hline Peso & 55,10 & 97,53 \\
\hline Altura de pie & 161,40 & 184,80 \\
\hline Altura sentado & 78,52 & 93,90 \\
\hline Altura de los ojos (sujeto sentado) & 70,68 & 84,70 \\
\hline Altura de la nuca (sujeto sentado) & 61,46 & 75,90 \\
\hline Altura de los hombros (sujeto sentado) & 47,92 & 64,93 \\
\hline Altura del codo asiento (sujeto sentado) & 20,00 & 29,46 \\
\hline Longitud hombro codo (sujeto sentado) & 30,10 & 37,00 \\
\hline Longitud codo muñeca (sujeto sentado) & 23,58 & 31,90 \\
\hline Anchura de hombros 2 (anchura bideltoidea, sujeto sentado) & 39,30 & 52,45 \\
\hline Anchura de hombros (sujeto sentado) & 23,60 & 44,90 \\
\hline Anchura entre codos (sujeto sentado) & 38,58 & 54,93 \\
\hline Anchura del codo (sujeto sentado) & 6,70 & 10,00 \\
\hline Anchura de caderas (sujeto sentado) & 32,00 & 41,90 \\
\hline Altura del poplíteo (sujeto sentado) & 40,10 & 52,70 \\
\hline Espesor del muslo (sujeto sentado) & 12,76 & 20,90 \\
\hline Altura de la rodilla (sujeto sentado) & 46,52 & 60,45 \\
\hline Longitud poplíteo trasero (sujeto sentado) & 36,64 & 49,57 \\
\hline Longitud rodilla trasero (sujeto sentado) & 46,40 & 60,00 \\
\hline Espesor del pecho P (profundidad del pecho, sujeto sentado) & 18,60 & 27,60 \\
\hline Espesor abdominal (sujeto sentado) & 17,68 & 26,90 \\
\hline Altura alcance vertical (sujeto sentado) & 117,92 & 141,19 \\
\hline Distancia nalga punta del pie (sujeto sentado) & 56,40 & 70,00 \\
\hline Distancia nalga pierna (sujeto sentado) & 92,22 & 109,73 \\
\hline Alcance punta dedo (sujeto sentado) & 73,20 & 82,87 \\
\hline Nota $*$ Medo & \\
\hline
\end{tabular}

Nota. ${ }^{*}$ Medidas en cm. 
En las siguientes figuras se puede observar el diseño de la butaca ergonómica.

Figura 2. Diseño ergonómico de butaca.

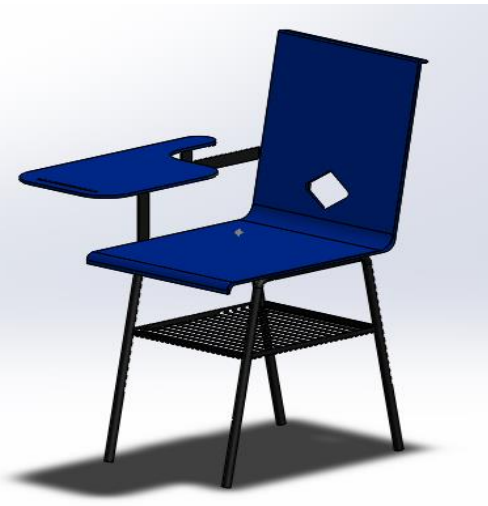

Figura 4. Longitud respaldo-paleta.

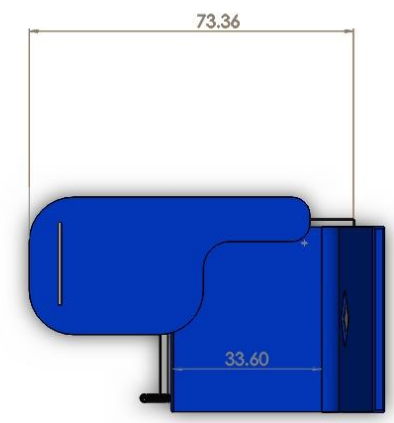

Figura 6. Ancho paleta.

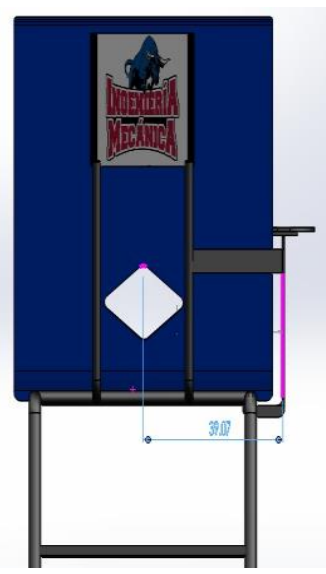

Figura 3. Ancho asiento.

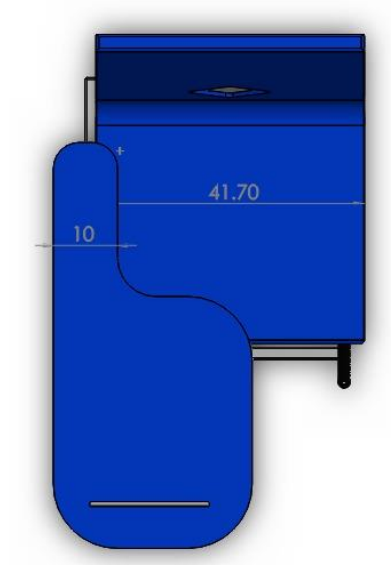

Figura 5. Altura paleta-asiento.

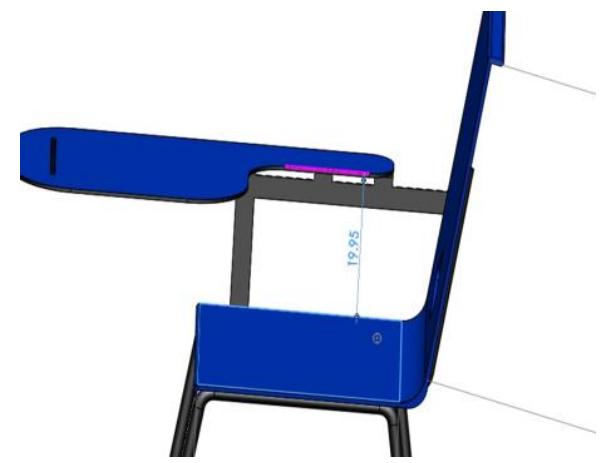

Figura 7. Largo asiento y respaldo.

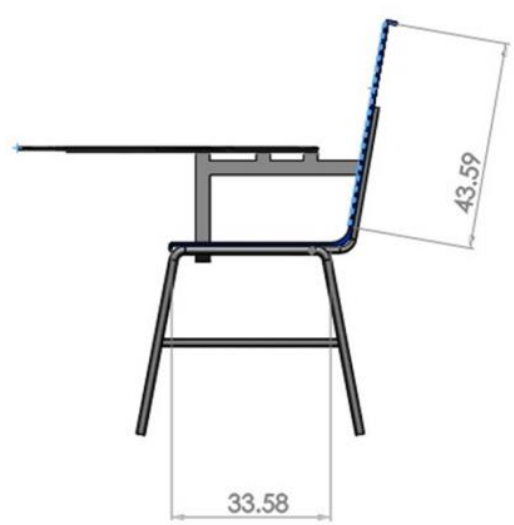


Figura 8. Altura asiento.

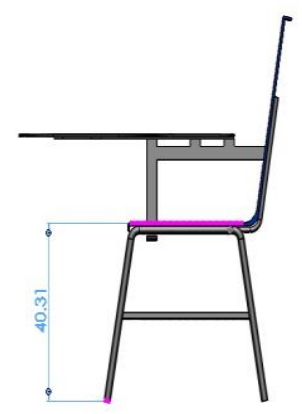

La investigación estuvo dirigida a 100 estudiantes de la carrera de ingeniería mecánica entre los 18 y 22 años de edad y se limitó al estudio antropométrico y el diseño de la butaca brindando la oportunidad de la fabricación futura.

\section{Conclusiones}

El correcto diseño de las butacas del aula es un factor muy importante que va a contribuir no solo a que los estudiantes puedan mantener durante la jornada escolar una postura adecuada y con ello reducir la fatiga y el dolor de espalda, sino también brindar comodidad si se está adaptado a las dimensiones físicas de los usuarios.

Esta investigación, consideró los elementos causantes de la incomodidad de los estudiantes de ingeniería mecánica de una universidad para sugerir un diseño que supere dichas causas. Adecuar las butacas a los estudiantes ayuda a disminuir el estrés y cansancio musculoesquelético, tener una buena postura y además facilita el desarrollo de las actividades, ya que permite dar comodidad a los alumnos y, a su vez, ayuda en su desempeño académico.

Es de vital importancia que en la actualidad se considere diseñar las estaciones de trabajo dentro de un esquema ergonómico que permita ser más productivos en las tareas cotidianas cuidando la salud física y mental de los usuarios. 


\section{Referencias}

Ávila, C., Prado, L., \& González, E. (2007). Dimensiones antropométricas de población latinoamericana. Universidad de Guadalajara.

Breve introducción a la antropometría física (2010, 10 de marzo). http://mooldesign.blogspot.com/2010/03/breve-introduccion-laantropometria.html\#: :text=La\%20antropometr\%C3\%ADa\%20aplicada\%20al\%20dise\%C3 \%B10,dimensiones\%20de\%20la\%20poblaci\%C3\%B3n\%2ometa

Cervantes-Soto, A. J., García-Saaib, A. R., Torres-Bonilla, X. Y., Castellanos-Magdaleno, G., \& Mercado-Mercado, G. (2019). Diagnóstico de lumbalgia en estudiantes universitarios del área de salud en Tepic, Nayarit. Medicina Legal de Costa Rica, 36(1), 43-53. https://www.scielo.sa.cr/scielo.php?script=sci arttext\&pid=S1409-00152019000100043

Medina, E., Illada, R., \& Domínguez, M. (2011). Problemas disergonómicos asociados al mobiliario escolar y al manejo de cargas en escolares de educación básica en planteles públicos y privados. Valencia, Venezuela. Comunidad y Salud, 9(1), 61-70. http://ve.scielo.org/scielo.php?script=sci arttext\&pid=S1690-32932011000100008

Párraga, R., \& García, T. (2014). Diseño ergonómico de aulas universitarias que permitan optimizar el confort y reducir la fatiga de estudiantes y docentes. Industrial Data, 17(2), 7-16. https://www.redalyc.org/articulo.oa?id=81640856002

Rodríguez, L. J., \& González, P. L. T. (2011). La evolución del mobiliario escolar. Técnica Industrial, 295, 64-69. https://www.tecnicaindustrial.es/la-evolucion-del-mobiliarioescolar/

Ruiz, M. R. O. (2008). Tablas antropométricas infantiles. Universidad Nacional de Colombia. https://repositorio.unal.edu.co/bitstream/handle/unal/7163/Ruiz Manuel\%2C tablas ant ropometricas.pdf?sequence $=1 \&$ isAllowed $=\mathrm{y}$

Unión General de Trabajadores (s.f). Riesgos relacionados con la ergonomía. Consultado el 13 de septiembre de 2019. https://riesgoslaborales.saludlaboral.org/portal-preventivo/riesgoslaborales/riesgos-relacionados-con-la-

hergonomia/ergonomia/\#: :text=La\%2ofunci\%C3\%B3n\%2oprincipal\%2ode\%2ola,las\%2op rimeras\%2oherramientas\%20que\%20invent\% 3 3\% 3

Vergara, M., \& Agost, M. J. (2015). Antropometría aplicada al diseño del producto. Universidad Jaume I, Servicio de comunicación y publicaciones. 

Anexo. Registro de mediciones.

\begin{tabular}{|c|c|c|c|c|c|c|c|c|c|c|c|c|c|c|c|c|c|c|c|c|c|c|c|c|c|}
\hline & $\begin{array}{l}0 \\
\vdots \\
0 \\
D_{1}^{2}\end{array}$ & 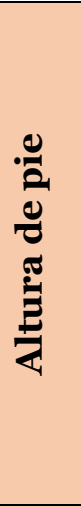 & 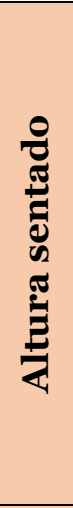 & 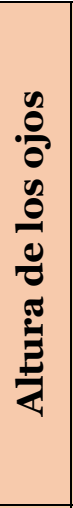 & 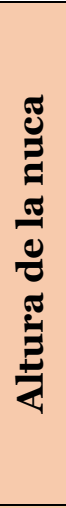 & 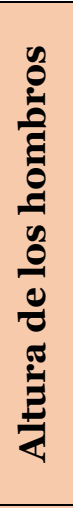 & 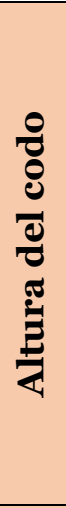 & 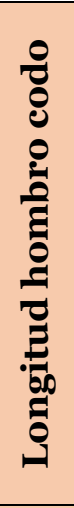 & 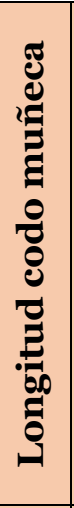 & 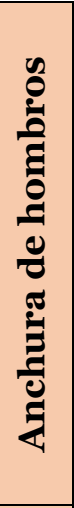 & 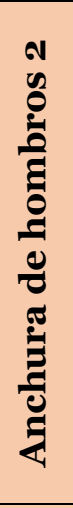 & 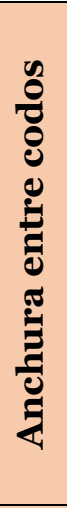 & 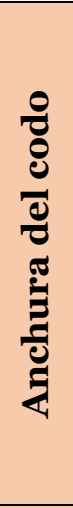 & 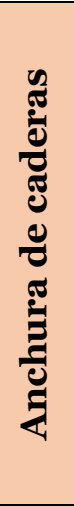 & 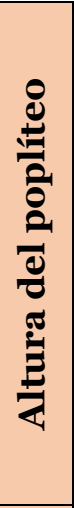 & 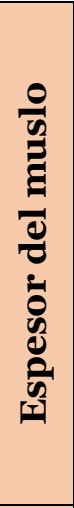 & 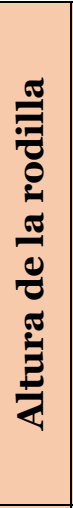 & 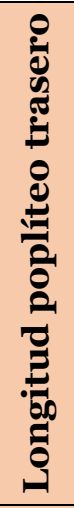 & 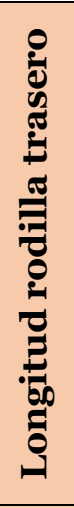 & 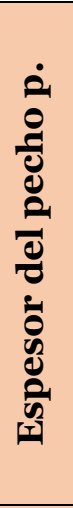 & 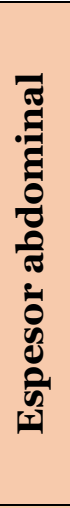 & 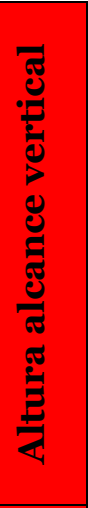 & 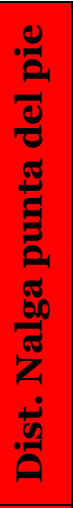 & 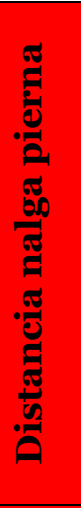 & 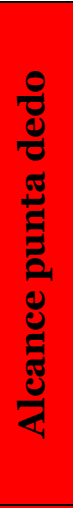 \\
\hline & 1 & & 3 & 4 & 5 & 6 & 7 & 8 & 9 & 10 & 11 & 12 & 13 & 14 & 15 & 16 & 17 & 18 & 19 & 20 & 21 & 22 & 23 & 24 & 25 \\
\hline 1 & & & 90 & 80 & 73 & 60 & 27 & 36 & 30 & 44 & 45 & 44 & 7 & 36 & 43 & 16 & 52 & 38 & 52 & 20 & 22 & 139 & 62 & 98 & 79 \\
\hline 2 & & & & 75 & 74 & 56 & 27 & 34 & 27 & 44 & 50 & 50 & 9 & 41 & 44 & 17 & 51 & 42 & 53 & 24 & 25 & 130 & 63 & 97 & 74 \\
\hline 3 & & & & 81 & & 64 & 30 & & 32 & 15 & 51 & -1 & 8 & & & & & 8 & & & 2 & & 71 & & \\
\hline 4 & & & & 73 & 67 & 8 & 24 & & 28 & 41 & & & & & & & & 4 & & & 4 & & & 8 & 124 \\
\hline 5 & & & & 73 & 6 & 55 & 20 & & 30 & 41 & & 4 & & & & & & 7 & & & 20 & & 66 & 3 & \\
\hline 6 & & & & 77 & 69 & 59 & 24 & 36 & 29 & & 51 & 49 & & & & & & 2 & & & 3 & & 70 & & 5 \\
\hline 7 & & & & 71 & 65 & 58 & 20 & 36 & 28 & & 54 & 54 & 7 & 36 & 41 & & & 47 & & & 23 & & 65 & & 71 \\
\hline 8 & & & & & 69 & 62 & 25 & 37 & 23 & 29 & 47 & 43 & 10 & 34 & 44 & 19 & & 43 & 56 & 21 & 21 & 135 & 66 & 98 & 73 \\
\hline 9 & 95 & & 93 & & 70 & 61 & 23 & 34 & 29 & 33 & 53 & 53 & 12 & 40 & 48 & & & 46 & 59 & 35 & 27 & 131 & 69 & 04 & 70 \\
\hline 10 & 7 & 177 & 90 & 85 & 73 & 64 & 26 & 36 & 29 & 29 & 46 & 46 & 10 & 36 & 47 & 18 & 46 & 43 & 59 & 21 & 21 & 123 & 69 & 5 & 59 \\
\hline 11 & 7 & & 91 & 85 & 71 & 62 & 26 & 33 & 31 & 29 & 47 & 50 & 10 & 35 & 45 & 20 & 58 & 42 & 55 & 21 & 23 & 132 & 65 & 102 & 70 \\
\hline 12 & 6 & & 90 & 80 & 70 & 63 & 29 & 35 & 30 & 33 & 46 & 48 & 8 & 33 & 46 & 17 & 53 & 41 & 52 & 21 & 20 & 137 & 62 & 99 & 74 \\
\hline 13 & 6 & & & 79 & 70 & 61 & 20 & 36 & 31 & 29 & 45 & 43 & 9 & 31 & 50 & & 60 & 44 & 54 & 22 & 21 & 131 & 64 & 104 & 70 \\
\hline 14 & 8 & & 85 & 75 & 65 & 58 & 24 & 35 & 30 & 27 & 48 & 53 & 10 & 37 & 46 & 18 & 56 & 40 & 55 & 24 & 23 & 120 & 65 & 96 & 62 \\
\hline 15 & 9 & & 95 & 86 & 64 & 66 & 27 & 36 & 30 & 31 & 50 & 50 & 10 & 37 & 41 & 21 & 62 & 45 & 59 & 24 & 25 & 135 & 69 & 109 & 69 \\
\hline 16 & 6 & & 9 & 80 & 68 & 61 & 28 & 34 & 30 & 26 & 42 & 39 & 9 & 33 & 41 & 17 & 53 & 42 & 49 & 18 & 19 & 124 & 59 & 94 & 63 \\
\hline 17 & & & & 82 & 76 & 64 & 30 & 35 & 28 & 31 & 47 & 52 & 8 & 39 & & & 58 & 46 & 57 & 26 & 26 & 139 & 67 & $\mathrm{OO}$ & 75 \\
\hline 18 & 74 & & & 73 & 65 & 58 & 27 & 34 & 29 & 30 & 44 & 47 & 9 & 38 & & 18 & 58 & 43 & 54 & 22 & 21 & 120 & 64 & 03 & 62 \\
\hline 19 & 7 & & & 73 & 66 & 58 & 22 & 38 & 32 & 46 & 39 & 48 & 8 & 40 & 56 & 16 & 46 & 56 & 60 & 24 & 22 & 137 & 70 & 110 & 79 \\
\hline & & & 84 & 72 & 67 & 56 & 27 & 36 & 39 & 44 & 56 & 46 & 9 & 42 & 40 & 16 & 54 & 42 & 55 & 27 & 27 & 129 & 65 & 99 & 73 \\
\hline 21 & 81 & 175 & 78 & 73 & 64 & 58 & 23 & 38 & 31 & 43 & 49 & 47 & 10 & 38 & 46 & 15 & 59 & 44 & 54 & 25 & 26 & 140 & 64 & 108 & 81 \\
\hline
\end{tabular}




\begin{tabular}{|c|c|c|c|c|c|c|c|c|c|c|c|c|c|c|c|c|c|c|c|c|c|c|c|c|c|}
\hline 22 & 72 & 170 & 87 & 75 & 65 & 9 & 24 & 33 & 28 & 27 & 46 & 48 & 8 & 36 & 48 & 16 & 56 & 44 & 51 & 22 & 23 & 128 & 61 & 100 & 69 \\
\hline 23 & 98 & 185 & 96 & 79 & 75 & 66 & 24 & 37 & 30 & 30 & 48 & 49 & 9 & 40 & 50 & 19 & 63 & 44 & 58 & 28 & 26 & 141 & 68 & 110 & \\
\hline 4 & 101 & 179 & 90 & 82 & 71 & 64 & 25 & 36 & 27 & 30 & 52 & 59 & 10 & 42 & 47 & 22 & 50 & 49 & 59 & 22 & 24 & 147 & 69 & 05 & \\
\hline 25 & 67 & 177 & 92 & 79 & 70 & 62 & 25 & 35 & 30 & 30 & 42 & 48 & 7 & 57 & 47 & 16 & 57 & 49 & 60 & 25 & 20 & 133 & 70 & & \\
\hline 26 & 81 & 183 & $\mathrm{O}$ & 77 & 72 & 63 & 26 & 37 & 31 & 32 & 46 & 47 & 10 & 37 & 43 & 13 & 56 & 47 & 5 & 23 & 20 & 141 & 65 & 05 & 78 \\
\hline 2 & 80 & 181 & 88 & 78 & 79 & 59 & 25 & 37 & 31 & 36 & 39 & 48 & 0 & 37 & 14 & 15 & 57 & 43 & 54 & 20 & 22 & 141 & 64 & 104 & 82 \\
\hline & 70 & 181 & 91 & 76 & 66 & 50 & 24 & 35 & 30 & 24 & 44 & 47 & 10 & 34 & 48 & 14 & 54 & 36 & 48 & 21 & 21 & 138 & 58 & 109 & 78 \\
\hline 29 & 51 & 166 & 36 & 73 & 67 & 58 & 25 & 33 & 26 & 27 & 41 & 41 & 8 & 39 & 45 & 15 & 53 & 44 & 52 & 19 & 15 & 122 & & & 64 \\
\hline 30 & 62 & 179 & 86 & 77 & 66 & 59 & 24 & 35 & 29 & 34 & 42 & 38 & 8 & 33 & 43 & 13 & 55 & 42 & 54 & 2 & 19 & 134 & & & 75 \\
\hline 31 & 55 & 161 & 88 & 77 & 69 & 61 & 30 & 31 & 26 & 27 & 42 & 38 & 7 & 38 & 41 & 12 & 49 & 43 & 54 & 25 & 18 & 128 & 64 & 93 & 67 \\
\hline 32 & 94 & 88 & 89 & 76 & 67 & 59 & 24 & 36 & 30 & 36 & 49 & 53 & 10 & 41 & 47 & 16 & 61 & 50 & 61 & 24 & 27 & 145 & 71 & 117 & 86 \\
\hline 33 & 64 & 68 & 83 & 73 & 61 & 54 & 25 & 30 & 29 & 27 & 46 & 45 & 9 & 32 & 44 & 14 & 54 & 33 & 46 & 20 & 19 & 127 & 56 & 93 & 73 \\
\hline 34 & 56 & 89 & 80 & 74 & 62 & 58 & 23 & 37 & 27 & 24 & 44 & 47 & 8 & 33 & 49 & 15 & 58 & 41 & 56 & 20 & 18 & 141 & 66 & 105 & 83 \\
\hline 35 & 76 & 9 & 86 & 71 & 62 & 54 & 29 & 32 & 26 & 26 & 42 & 46 & 9 & 38 & 47 & 17 & 55 & 39 & 55 & 21 & 22 & 24 & 65 & 8 & 70 \\
\hline 36 & 65 & 165 & 78 & 65 & 54 & 47 & 26 & 31 & 25 & 25 & 45 & 43 & 9 & 35 & 43 & 14 & 54 & 39 & 49 & 21 & 20 & 126 & 59 & 96 & 79 \\
\hline & 79 & 159 & 81 & 71 & 63 & 56 & 25 & 31 & 23 & 42 & 48 & 55 & 11 & 39 & 42 & 15 & 50 & 42 & 50 & 25 & 26 & 117 & 50 & & 61 \\
\hline 38 & 60 & 173 & 80 & 74 & 65 & 60 & 26 & 34 & 30 & 32 & 43 & 48 & 9 & 40 & 50 & 8 & 57 & 45 & 56 & 2. & 20 & 140 & 56 & 100 & 80 \\
\hline 39 & 59 & & 88 & 77 & 64 & 59 & 25 & 19 & 28 & 29 & 43 & 43 & & 3 & & 16 & 54 & 44 & 50 & $C$ & 20 & 135 & 50 & & 76 \\
\hline $4 c$ & 60 & & 92 & 76 & 69 & 57 & 23 & 34 & 20 & 29 & 43 & 39 & & 36 & 50 & & 52 & 38 & 46 & 20 & 19 & 30 & & 89 & 73 \\
\hline & 7 & & 88 & 78 & 73 & 6 & & 35 & 2 & & 47 & 50 & & 38 & 44 & & 55 & 40 & 52 & & 23 & & 6 & & 68 \\
\hline 4 & 9 & & 90 & 82 & 76 & 65 & 28 & 33 & 28 & 26 & 47 & 56 & & 40 & 55 & 20 & 57 & 40 & 55 & & 26 & 36 & & & 71 \\
\hline 43 & 58 & 168 & 90 & 80 & 73 & 6 & 25 & 33 & 30 & 27 & 4. & 41 & & 32 & 41 & & 52 & 42 & 54 & & 19 & 36 & 64 & & \\
\hline 44 & 80 & 70 & 86 & 77 & 70 & 66 & 28 & 34 & 28 & 24 & 48 & 51 & & 41 & 43 & 16 & & 44 & 56 & 24 & 22 & 135 & 66 & 99 & 69 \\
\hline
\end{tabular}

